Physics

Physics Research Publications

\title{
Parton cascade and coalescence
}

D. Molnar 


\section{Parton cascade and coalescence}

Denes Molnarabc

aPhysics Department, Ohio State University

191 West Woodruff Avenue, Columbus, OH 43210, USA

${ }^{\mathrm{b}}$ Physics Department, Purdue University

525 Northwestern Avenue, West Lafayette, IN 47907, USA

${ }^{\mathrm{c}} \mathrm{RIKEN} / \mathrm{BNL}$ Research Center

Brookhaven National Laboratory, Upton, NY 11973-5000, USA

This is a review of the parton cascade approach and its implications to parton coalescence at RHIC.

\section{Introduction}

Recent data from $A u+A u$ reactions at $\sqrt{s_{N N}}=130$ and $200 \mathrm{GeV}$ from RHIC show striking difference between baryons and mesons in the intermediate transverse momentum region $2<p_{\perp}<5 \mathrm{GeV}$. First, elliptic flow scales with constituent quark number[1-5]. The flow per constituent quark as a function of transverse momentum per constituent quark, $v_{2}^{\text {hadron }}\left(p_{T} / n\right) / n$ ( $n=2$ for mesons, 3 for baryons), is a universal function for all hadron species (within experimental uncertainties). Second, nuclear suppression is weaker ( $R_{A A}$ is larger) for baryons than for mesons[1,2,6,7,4,8,9]. The most promising mechanism proposed to explain both phenomena is parton coalescence[10-17].

In the coalescence model, mesons form from a quark and antiquark, while baryons from three quarks or three antiquarks (contributions from higher Fock states are small [18]). Most versions of the model are based on variants of the simple "coalescence formula" [19, $20,13,14,16]$

$$
\frac{d N_{h a d}(\vec{p})}{d^{3} p}=g_{M} \int \prod_{i}\left[d^{3} x_{i} d^{3} p_{i} f_{i}\left(\vec{p}_{i}, \vec{x}_{i}\right)\right] W_{h a d}\left(\left\{\Delta \vec{x}_{i j}\right\},\left\{\Delta \vec{p}_{i j}\right\}\right) \delta^{3}\left(\vec{p}-\sum_{i} \vec{p}_{i}\right)
$$

that gives the hadron spectra in terms of the constituent phasespace distributions $f_{i}$ on a 3D spacetime hypersurface and the Wigner-transform of hadron wave functions $W$. (Arbitrary 3D hypersurfaces[19,20] and quark correlations[21] are straightforward to accommodate.) This simple approach can reproduce the particle spectra at RHIC quite well, with coalescence hadronization from a thermalized quark-antiquark plasma[13,14] and an additive fragmentation contribution of quenched high- $p_{T}$ jets[22]. The scaling of elliptic flow with constituent quark number was also explained in this framework[15,16].

Nevertheless, several important questions are still open. At low $p_{T}$, the coalescence formula violates[15,16] unitarity. The yield in a given coalescence channel scales quadrat- 
ically/cubically with constituent number, moreover, the same constituent contributes to several channels (including fragmentation in certain schemes). Energy conservation is only approximate because of the on-shell treatment and neglect of binding energies. Also, though the final entropy is comparable to the initial one (due to hadron decays) [23], it is problematic that entropy does decrease temporarily during the coalescence process.

Most importantly, the simple parametrizations assumed for the quark phase space distributions are inconsistent with dynamical models, such as parton cascades or hydrodynamics. Spatial inhomogeneities and dynamical phasespace correlations distort coalescence predictions in a crucial way. In particular, the observed quark number scaling becomes highly nontrivial [24], and a large baryon/meson ratio enhancement is difficult to achieve[17].

Below I review and illustrate the difficulties, using one of the main dynamical approaches, covariant parton transport theory.

\section{Covariant parton transport theory}

Covariant parton transport theory is the incoherent, particle (short-wavelength) limit of quantum-chromodynamics. Its main advantage is that it is applicable out of equilibrium and models freezeout self-consistently. However, it cannot describe phase transitions (without coupling to classical fields).

One of the simplest but nonlinear forms of Lorentz-covariant Boltzmann transport theory involves on-shell phase space densities $\left\{f_{i}(x, \vec{p})\right\}$ that evolve with elastic $2 \rightarrow 2$ $[25-29]$ and inelastic $2 \rightarrow 2[30]$ rates as

$p_{1}^{\mu} \partial_{\mu} f_{1, i}=S_{i}\left(x, \vec{p}_{1}\right)+\frac{1}{16 \pi^{2}} \sum_{j k \ell} \iiint_{234}\left(f_{3, k} f_{4, \ell} \frac{g_{i} g_{j}}{g_{k} g_{\ell}}-f_{1, i} f_{2, j}\right)\left|\overline{\mathcal{M}}_{12 \rightarrow 34}^{i j \rightarrow k \ell}\right|^{2} \delta^{4}\left(p_{1}+p_{2}-p_{3}-p_{4}\right)$.

$|\overline{\mathcal{M}}|^{2}$ is the polarization averaged scattering matrix element squared, the integrals are shorthands for $\int \equiv \int d^{3} p_{a} /\left(2 E_{a}\right), g_{i}$ is the number of internal degrees of freedom for species $i$, while $f_{a, i} \equiv f_{i}\left(x, \vec{p}_{a}\right)$. The source functions $\left\{S_{i}(x, \vec{p})\right\}$ specify the initial conditions.

Eq. (2) could in principle be extended for bosons and/or for inelastic processes, such as $g g \leftrightarrow g g g$ [31,32]. However, with the new nonlinearities these extensions introduce, it is very challenging to maintain Lorentz covariance numerically at opacities expected at RHIC. Off-shell variants of parton transport also exist, such as the VNI/b model[33].

Based on the local mean free path $\lambda(x, s) \equiv 1 /[n(x) \sigma(s)]$, the theory naturally interpolates between ideal hydrodynamics $(\lambda \rightarrow 0)$ and free streaming $(\lambda \rightarrow \infty)$. The most relevant quantity that characterizes the whole evolution is the transport opacity $\chi \equiv \int d z \rho(z) \sigma_{t r}[28]$, which is the average number of collisions per parton multiplied by the ratio of the transport and total cross sections (the efficiency of momentum transfer in a single collision). In a near-equilibrium situation, these parameters can be related to transport coefficients, such as the shear viscosity or diffusion constants.

Transport theory provides important information about the properties of the partonic medium created at RHIC. The large elliptic flow observed[34-36] indicates a stronglyinteracting, opaque parton system[28], with $d N_{g} / d \eta(b=0) \times \sigma_{g g} \approx 1000 \times 45 \mathrm{mb}$, about 15 times above the perturbative estimate (see Fig. 1a). These conditions are still different from an ideal fluid because dissipation reduces elliptic flow by $30-50 \%$ relative to the 


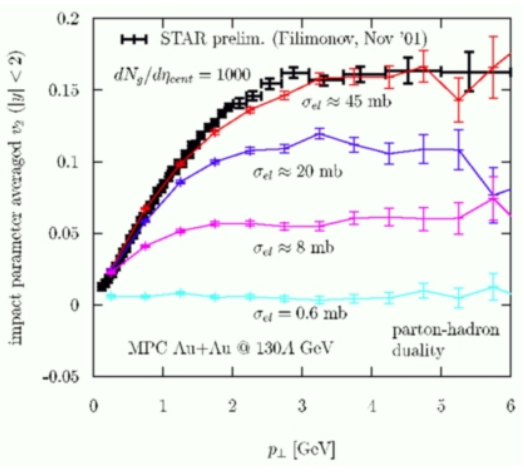

a)

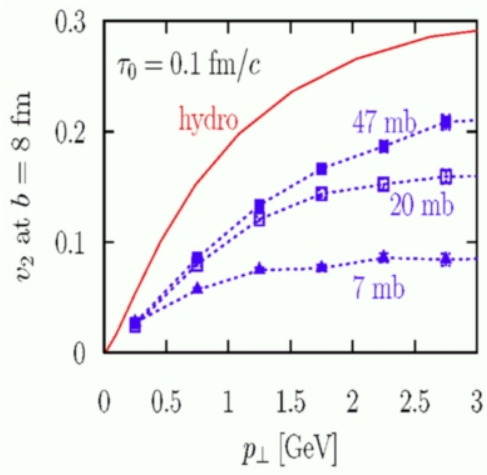

b)

Figure 1. Results for $A u+A u$ at $\sqrt{s}=200 A \mathrm{GeV}$ at RHIC with $b=8$ fm from MPC[40]. a) Transport opacity dependence of elliptic flow as a function of $p_{T}$ from covariant transport theory (from [28]); b) comparison of elliptic flow as a function of $p_{T}$ from covariant transport theory and ideal hydrodynamics (from [29]).

ideal fluid limit [29] (Fig. 1b). At such high opacities, the shear viscosity of the parton plasma $\eta \sim s \lambda T / 5$ [37] (estimated from kinetic theory) is indeed very small, close to the conjectured lower bound $\eta_{\min }=s /(4 \pi)$ [38] ( $s$ is the entropy density). However, the small viscosity is compensated by large gradients in heavy-ion collisions, resulting in significant dissipative effects. Estimates based on viscous (Navier-Stokes) hydrodynamics also support this conclusion[39].

Though even the large opacities at RHIC are insufficient to equilibrate charm, a large charm quark elliptic flow is still expected above $p_{T} \sim 2-3 \mathrm{GeV}$ (Fig. 2) from several studies (covariant transport predictions from MPC[30,40], reinforced by calculations from AMPT[41] and also a Fokker-Planck approach[42]). It is very exciting that preliminary indirect data from RHIC do indicate a nonzero charm elliptic flow $[43,44]$. These data are on the elliptic flow of electrons from $D$ meson decays $D^{(*)} \rightarrow K \nu e$, which based on model studies follows closely the $D$ meson elliptic flow $[45,41]$ (see Fig. 2b).

At high $p_{T}$ covariant transport shares a lot of similarity with parton energy loss models[22], except that $2 \rightarrow 2$ transport gives incoherent, elastic energy loss. However, at the high opacities at RHIC the dynamics is much richer. Not only energy loss, but significant energy gain is also possible in multiple collisions[46]. This "plasma push" process, a shadow of near-hydrodynamic behavior at low $p_{T}$, plays a role even at $p_{T} \sim 10 \mathrm{GeV}$, and is the reason why elliptic flow from the transport decreases very slowly at high $p_{T}$.

\section{Dynamical effects and elliptic flow scaling from coalescence}

To illustrate the effect of the dynamics on elliptic flow scaling from coalescence, it is useful to first ignore variations of phasespace distributions on length and momentum scales corresponding to a typical hadron $(\sim 1 \mathrm{fm}$ and $\sim 200 \mathrm{MeV})$. In this case[24], 

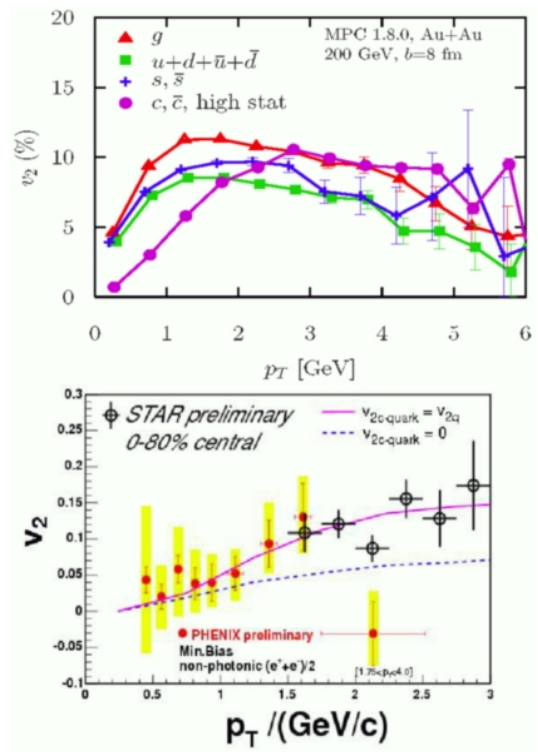

a)

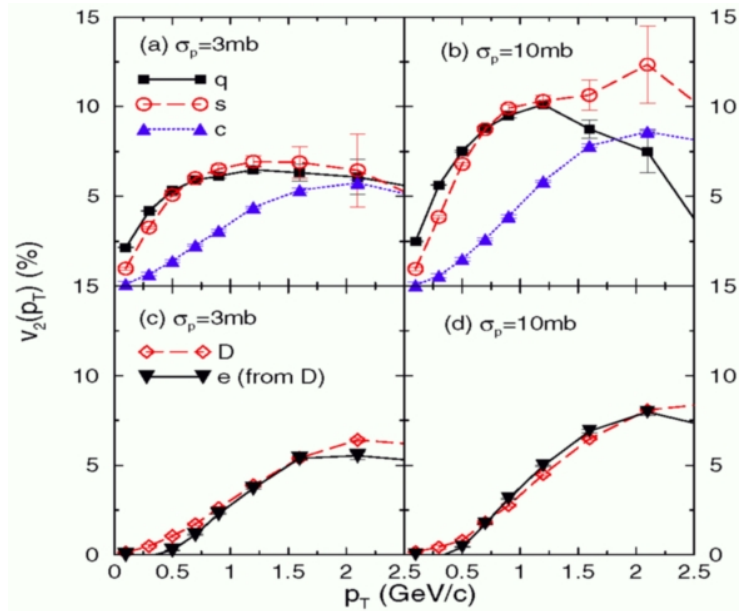

b)

Figure 2. a) Top panel: parton species dependence of elliptic flow in $A u+A u$ at $\sqrt{s_{N N}}=$ $200 \mathrm{GeV}$ at RHIC as a function of $p_{T}$, for $b=8 \mathrm{fm}$ and $\approx 7$ times perturbative opacities, from the covariant MPC model (from [30]); bottom panel: preliminary non-photonic electron $v_{2}\left(p_{T}\right)$ data from RHIC (from [43]). b) Top panels: parton $v_{2}\left(p_{T}\right)$ for minimumbias $A u+A u$ collisions at $\sqrt{s_{N N}}=200 \mathrm{GeV}$ from the AMPT model; bottom panels: comparison of $D$ meson $v_{2}$ and electron $v_{2}$ from $D$ decays (from [41]).

$W \sim \delta^{3}\left(\Delta \vec{x}_{i j}\right) \delta^{3}\left(\Delta \vec{p}_{i j}\right)$ and the phasespace distributions of mesons and baryons from coalescence $(\alpha \beta \rightarrow M, \alpha \beta \gamma \rightarrow B)$ are

$$
\begin{aligned}
f_{B}(x, \vec{p}) & =\frac{(2 \pi)^{3} g_{B}}{g_{\alpha} g_{\beta} g_{\gamma}} f_{\alpha}(x, \vec{p} / 3) f_{\beta}(x, \vec{p} / 3) f_{\gamma}(x, \vec{p} / 3) \\
f_{M}(x, \vec{p}) & =\frac{(2 \pi)^{6} g_{M}}{g_{\alpha} g_{\beta}} f_{\alpha}(x, \vec{p} / 2) f_{\beta}(x, \vec{p} / 2)
\end{aligned}
$$

$g$ is the degeneracy of the particle (spin and color), and I considered constituent quarks of (roughly) equal mass, which share the total hadron momentum (roughly) equally. (In hadrons that also contain a heavy quark, e.g., $D$ mesons or the $\Lambda_{c}$, the heavy quark carries most of the momentum [16].)

Assume for simplicity that all quarks have identical phase space distributions

$f_{q}\left(x, \vec{p}_{T}, y=0\right) \equiv n_{q}\left(x, p_{T}\right)\left[1+\sum_{n=1}^{\infty} 2 v_{n, q}\left(x, p_{T}\right) \cos (n \phi)\right]$

where $n_{q}$ is the local density of quarks with transverse momentum (magnitude) $p_{T}$. For 
small local anisotropies

$\left|v_{2, q}\right| \ll 1, \quad\left|v_{k, q} v_{l, q}\right| \ll\left|v_{2, q}\right|$,

the usual assumption to obtain elliptic flow scaling, the hadron elliptic flows from (3) are

$$
\begin{aligned}
v_{2, M}\left(p_{T}\right) & \approx \frac{2\left\langle n_{q}^{2}\left(x, p_{T} / 2\right) v_{2, q}\left(x, p_{T} / 2\right)\right\rangle_{x}}{\left\langle n_{q}^{2}\left(x, p_{T} / 2\right)\right\rangle_{x}} \\
v_{2, B}\left(p_{T}\right) & \approx \frac{3\left\langle n_{q}^{3}\left(x, p_{T} / 3\right) v_{2, q}\left(x, p_{T} / 3\right)\right\rangle_{x}}{\left\langle n_{q}^{3}\left(x, p_{T} / 3\right)\right\rangle_{x}}
\end{aligned}
$$

with $\langle A(x)\rangle_{x} \equiv \int d^{3} x A(x)$ (spatial average). Though each small spatial region with its own local quark elliptic flow contributes a local hadron elliptic flow that scales with quark number, $v_{2, \text { had }}\left(x, p_{T}\right) \approx n v_{2, q}\left(x, p_{T} / n\right)$, quark number scaling does not follow in general for the measured (spatially averaged) elliptic flow, i.e., $v_{2, h a d}\left(p_{T}\right) \neq n v_{2, q}\left(p_{T} / n\right)$. This is because constituent phase space densities appear nonlinearly, raised to second and third power.

Constituent quark scaling arises only for special classes of quark phase space distributions. Two classes where the spatial dependence in (3) cancels out trivially are i) when the local density is spatially uniform $n\left(x, p_{T}\right)=n\left(p_{T}\right)$ (as assumed in [15]); and ii) when the local flow anisotropy is spatially uniform $v_{2}\left(x, p_{T}\right)=v_{2}\left(p_{T}\right)$ (the assumption in [14]).

Unfortunately, neither of the two cases is realistic. Dynamical models yield spatially nonuniform local densities and nonuniform flow anisotropies. Local density variations have been studied in connection with the pion interferometry puzzle from hydrodynamics[47] and several transport models[48-50] (Fig. 3a is an example from covariant parton transport). Local flow anisotropies are also spatially nonuniform as can be seen in Fig. $3 \mathrm{~b}$ that shows (momentum) azimuthal angle $\phi$ distributions at midrapidity for $1<p_{T}<2 \mathrm{GeV}$, for $A u+A u$ at top RHIC energy, $b=8 \mathrm{fm}$, averaged over four wedges in the transverse coordinate plane $\varphi_{x} \in[k \pi / 8,(k+1) \pi / 8]$, with $k=0,1,2,3$ [24]. Clearly, partons emitted from in-plane regions move predominantly in-plane and, therefore, have positive elliptic flow $v_{2}>0$; while partons coming from out-of-plane regions move in out-of-plane direction and have negative $v_{2}<0$. This is very similar to the hydrodynamic expectation[51] based on radially boosted fireballs.

Fig. $3 \mathrm{~b}$ also demonstrates that the condition of small local anisotropies (5), which is necessary for general quark number scaling, is not satisfied either. Instead of small harmonic modulations over a uniform background, the distributions are strongly peaked because high- $p_{T}$ particles can only escape from a surface layer of the reaction region. In this case, local anisotropies from coalescence follow a unique power-law scaling [24] $\left|v_{\text {had }}\left(p_{T}\right)\right| \simeq\left|v_{2, q}\left(p_{T} / n\right)\right|^{1 / n}$, instead of the linear one derived in [15].

Therefore, quark number scaling of elliptic flow requires a highly nontrivial fortuitous interplay between variations in local parton density and nonlinear couplings between large local flow coefficients. Several classes of phase space distributions have been explored recently $[52,53]$ and solutions have been found that scale approximately.

One particularly simple example[53], with large local flow anisotropies, is when $N$ partons move along the $\phi=0$ axis and $(1-a) N$ partons move along $\phi=\pi / 2(a \ll 1)$. If the local volumes for both components are identical, the hadron yields are $N_{\text {had }} \propto V(N / V)^{n}+$ 


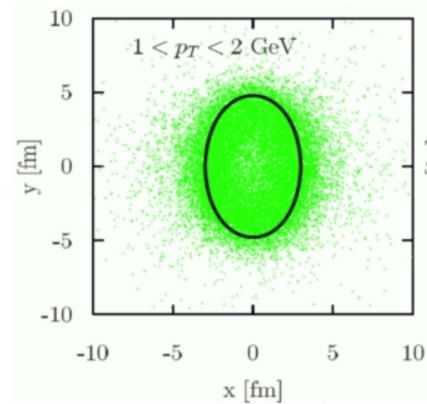

a)

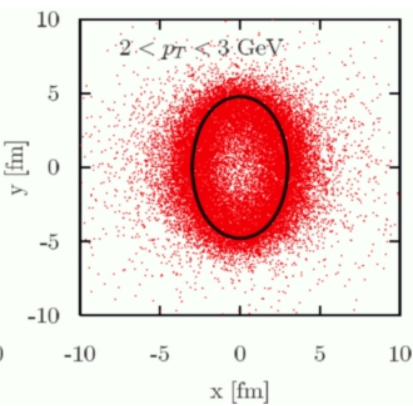

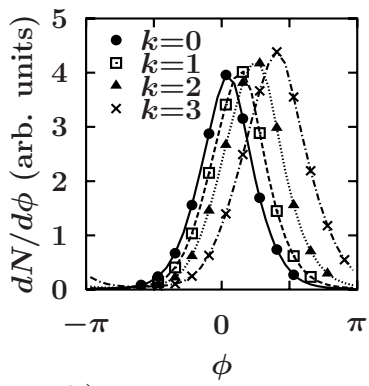

b)

Figure 3. Results from MPC[40] for $A u+A u$ at $\sqrt{s_{N N}}=200 \mathrm{GeV}$ with $b=8 \mathrm{fm}$. a) Transverse positions of partons at freezeout as a function of $p_{T}$, for final rapidities $|y|<2$ (scatter plot). The ellipses guide the eye. b) $\phi$ distributions at midrapidity for $1<p_{T}<2$ $\mathrm{GeV}$, averaged over four wedges in the transverse plane $\varphi_{x} \in[k \pi / 8,(k+1) \pi / 8], k=0 \ldots 3$ (from [24]).

$V((1-a) N / V)^{n}$, and the elliptic flows do scale $v_{2}=\left[(N / V)^{n}-((1-a) N / V)^{n}\right] /\left[(N / V)^{n}+\right.$ $\left.((1-a) N / V)^{n}\right] \approx n a / 2$. However, it is easy to show that scaling crucially depends on the assumption of identical volumes. Different local volumes, $V$ and $V^{\prime}$, give hadron yields $N_{\text {had }} \propto V(N / V)^{n}+V^{\prime}\left((1-a) N / V^{\prime}\right)^{n}$ and elliptic flows that do not scale, $v_{2}=$ $\left.\left.\left[\left(V / V^{\prime}\right)^{n-1}-(1-a)^{n}\right] /\left[\left(V / V^{\prime}\right)^{n-1}+(1-a)^{n}\right] \approx\left[\left(V / V^{\prime}\right)^{n-1}-1+n a\right)\right] /\left[\left(V / V^{\prime}\right)^{n-1}+1+n a\right)\right]$. This underscores the importance of going beyond simple parameterizations and using real dynamical models to study parton coalescence in heavy-ion collisions.

It is important to realize that quark number scaling from coalescence necessitates nonequilibrium dynamics[24]. For thermal constituent distributions, coalescence reduces to statistical hadronization and, therefore, momentum anisotropies in that case can only depend on particle mass, and not quark number. This is so even for nonequilibrium quark distributions that are of the form $f(x, \vec{p})=g\left(p_{\mu} u^{\mu}(x), x\right)$. Therefore, besides the breakdown of hydrodynamic behavior, quark number scaling indicates a marked departure from pure "flow-like" space-momentum correlations as well.

\section{Dynamical coalescence approach}

One way to study realistic dynamical effects is to combine nonequilibrium parton transport theory and coalescence [17]. Such an approach requires overcoming the limitations of (1) and proper book-keeping to ensure that each parton participates in one hadronization channel (no multiple counting).

The simple coalescence formula (1) relies on the assumption that interactions between quarks that are not in the same hadron cease suddenly on some 3D hypersurface. However, self-consistent freezeout from transport approaches gives diffuse 4D freezeout distributions [48-50] that cannot be well approximated with a hypersurface. The coalescence formalism was extended to such a case by Gyulassy, Frankel and Remler (GFR) in [54] (for weakly-bound states). Their result is the same as (1), except that 
the weight $W$ is evaluated using the freezeout coordinates $(t, \vec{x})$ of constituents. When taking $\Delta \vec{x}$, the earlier particle needs to be propagated to the time of the later one, e.g., $\Delta \vec{x}_{12}=\vec{x}_{1}-\vec{x}_{2}+\left(t_{2}-t_{1}\right) \vec{v}_{1}$ if $t_{1}<t_{2}$. The origin of this correction is that a weak bound state survives only if none of its constituents have any further interactions. For baryons, the generalization involves propagation to the latest of the three freezeout times.

For proper book-keeping, it is common in transport models[55] to utilize box Wigner functions $W=\prod_{i, j} \Theta\left(x_{m}-\left|\Delta \vec{x}_{i j}\right|\right) \Theta\left(p_{m}-\left|\Delta \vec{p}_{i j}\right|\right)$. This way (1) has a simple interpretation: if $W=1$ (and the quantum numbers match) the hadron is formed, otherwise it is not $(W=0)$. If several coalescence final states exist for a given constituent, one is chosen randomly. Partons that do not find a coalescence partner would propagate "freely" and are therefore fragmented independently.

Fig. 4 shows pion and proton elliptic flow at RHIC for $A u+A u$ at $\sqrt{s_{N N}}=200 \mathrm{GeV}$ with $b=8 \mathrm{fm}$ and $\approx 7$ times perturbative opacities $\left(\sigma_{g g \rightarrow g g}=10 \mathrm{mb}\right)$, from the dynamical coalescence approach[17] based on the covariant transport model MPC[40]. The elliptic flow of direct pions and protons from coalescence (left panel) is somewhat smaller than the parton flow scaled by constituent quark number. The difference is due to genuine dynamical effects that are much larger than few-percent corrections nonlinear in $v_{2}$ or those due to higher-order flow anisotropies[15]. Nevertheless, in view of the discussion in the previous Section, it is quite remarkable that the end result is only a modest $\sim 20 \%$ and $\sim 30 \%$ flow reduction for mesons and baryons.

On the other hand, fragmentation contributions sharply reduce the hadron $v_{2}$ as shown in the right panel in Fig. 4. Unlike the flow amplification from coalescence[15], fragmentation smears out the anisotropy because hadrons from the parton shower are not fully collinear (nonzero jet width). Because only a smaller fraction of parton finds a coalescence partner, a large fraction fragments, including essentially all partons above $p_{T}>2.5 \mathrm{GeV}$, as can be seen in Fig. 5a. Coalescence does become more and more important at low $p_{T}$ but it involves only about one in every three partons.
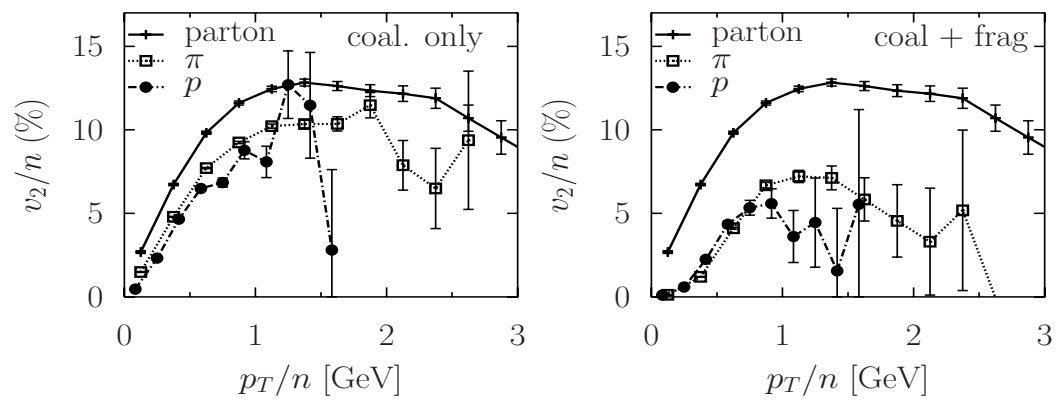

Figure 4. Quark number scaled elliptic flow from MPC[40] as a function of $p_{T}$ for pions (open squares) and protons (filled circles) in $A u+A u$ at $\sqrt{s}=200 A \mathrm{GeV}$ at RHIC with $b=8$ and $\sigma_{g g}=10 \mathrm{mb}$, with hadronization via combined coalescence and fragmentation (right), and for primary hadrons (without decays) from coalescence (left). The constituent $v_{2}\left(p_{T}\right)$ is also shown (solid lines). Taken from [17]. 


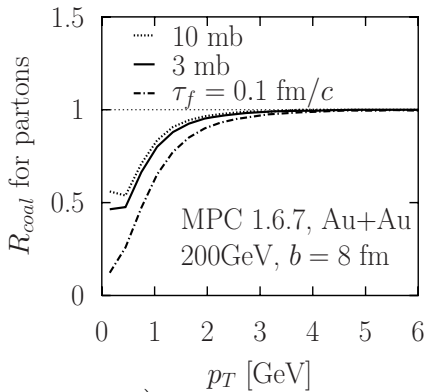

a)

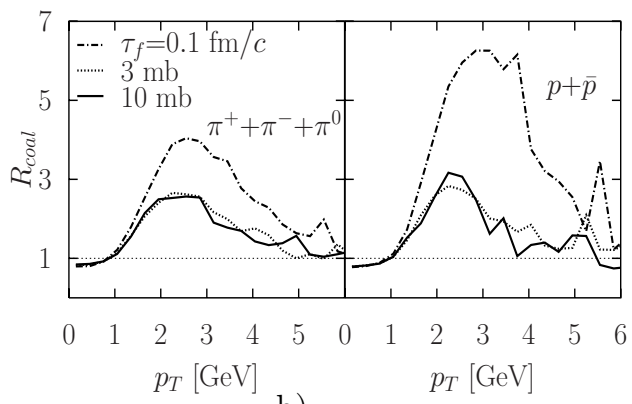

b)

Figure 5. Results from MPC for $A u+A u$ at $\sqrt{s}=200 A \mathrm{GeV}$ at RHIC with $b=8 \mathrm{fm}$ for $\sigma_{g g}=3 \mathrm{mb}$ (dotted) and $10 \mathrm{mb}$ (solid), or immediate freezeout at $\tau=0.1 \mathrm{fm} / \mathrm{c}$ (dasheddotted line). Taken from [17]. a) Fraction of partons that fragment independently as a function of $p_{T} ; \mathrm{b}$ ) pion and proton enhancement from coalescence as a function of $p_{\perp}$.

Despite the large fragmentation component, pion and proton $v_{2}$ still scale approximately relative to each other - up to small differences of $\sim 10 \%$, perhaps up to $30 \%$, which could be tested against high-precision Run-4 data from RHIC (better theory accuracy in the $p_{T} / n>1-1.5 \mathrm{GeV}$ region is certainly desirable). However, quantitative interpretation of the data becomes more complicated because the scaled hadron flows underpredict the real parton $v_{2}\left(p_{T}\right)$ by factor of 2 or more.

Dynamical effects on the baryon/meson ratio are much more striking as can be seen in Fig. 5b. Here the yield enhancement from coalescence is characterized by $R_{\text {coal }}$, the ratio of the final spectra with hadronization via combined coalescence and fragmentation to that with hadronization via fragmentation only. Parton coalescence enhances both pion and proton yields, and hence $R_{A A}$, by as much as a factor of three in the "coalescence window" $[11,15] 1.5<p_{T}<4.5 \mathrm{GeV}$. The additional hadron yield comes dominantly from partons with $0.5<p_{T}<2 \mathrm{GeV}$ (see Fig. 5a). The enhancement, however, is about the same for both protons and pions, and hence $p / \pi$ stays close to the value in $p+p$ collisions. Even for a (rather unrealistic) scenario with immediate freezeout on the formation $\tau=0.1 \mathrm{fm} / c$ hypersurface, which results in very high parton densities, the $p / \pi$ ratio is enhanced relative to $p+p$ only $1.5-1.7$ times, much less than the factor $\approx 3$ seen at RHIC at this centrality[6].

\section{Conclusions}

Parton coalescence is a promising approach to explain the striking baryon-meson difference in the $A u+A u$ data at intermediate $2<p_{T}<5 \mathrm{GeV}$ from RHIC. Studies based on simple parameterizations are quite successful but are inconsistent with dynamical models. A dynamical coalescence approach based on parton transport theory does confirm that coalescence is an important hadronization channel at RHIC. However, the dynamics significantly affects elliptic flow scaling and baryon/meson ratios. Until a reliable approach can successfully reproduce the data, "the jury is still out" regarding coalescence.

Clearly, further studies are needed. The $2 \rightarrow 2$ dynamics considered could be an 
oversimplification, or the formalism based on weakly-bound states may not apply well to the QCD coalescence process. Long-range correlations could make coalescence nonlocal in phase-space (unlike (1)), or help "optimally match" coalescence partners to maximize the yields. Precise experimental data on deviations from quark number scaling, flavor dependence of observables, and the transition to the pure fragmentation regime at high $p_{T}$ will provide additional tests of coalescence models and further insights into the dynamics at intermediate $p_{T}$.

\section{Acknowledgments}

Computer resources by the PDSF/LBNL and the hospitality of INT Seattle and the RIKEN/BNL Research Center, where part of this work was done, are gratefully acknowledged. This work was supported by DOE grant DE-FG02-01ER41190.

\section{REFERENCES}

1. M. Oldenburg [STAR Collaboration], nucl-ex/0510026; M. Konno [PHENIX Collaboration], nucl-ex/0510022; see also talks by O. Barannikova, X. Cai, D. Pal, H. Masui, and G. Wang.

2. P. Sorensen [STAR Collaboration], J. Phys. G 30 (2004) S217; J. Adams et al. [STAR Collaboration], Phys. Rev. Lett. 92 (2004) 052302.

3. S. S. Adler et al. [PHENIX Collaboration], Phys. Rev. Lett. 91 (2003) 182301.

4. H. b. Zhang [STAR Collaboration], nucl-ex/0403010.

5. J. Adams et al. [STAR Collaboration], nucl-ex/0504022.

6. S. S. Adler et al. [PHENIX Collaboration], Phys. Rev. Lett. 91 (2003) 172301; Phys. Rev. C 69 (2004) 034909.

7. H. Long [STAR Collaboration] J. Phys. G 30 (2004) S193.

8. J. Adams et al. [STAR Collaboration], Phys. Lett. B 612, 181 (2005).

9. S. S. Adler et al. [PHENIX Collaboration], Phys. Rev. C 72, 014903 (2005).

10. T. S. Biro, P. Levai and J. Zimanyi, Phys. Lett. B 347, 6 (1995); P. Csizmadia et al., J. Phys. G 25, 321 (1999).

11. S. A. Voloshin, Nucl. Phys. A 715 (2003) 379.

12. R. C. Hwa and C. B. Yang, Phys. Rev. C 66 (2003) 025205; ibid. 67 (2003) 034902; Phys. Rev. C 70, 024904 (2004).

13. V. Greco, C. M. Ko and P. Levai, Phys. Rev. Lett. 90 (2003) 202302; Phys. Rev. C 68 (2003) 034904;

14. R. J. Fries et al., Phys. Rev. Lett. 90 (2003) 202303; Phys. Rev. C 68 (2003) 044902;

C. Nonaka, R. J. Fries and S. A. Bass, Phys. Lett. B 583 (2004) 73.

15. D. Molnar and S. A. Voloshin, Phys. Rev. Lett. 91 (2003) 092301; D. Molnar, J. Phys. G 30 (2004) S235.

16. Z. w. Lin and D. Molnar, Phys. Rev. C 68 (2003) 044901.

17. D. Molnar, Acta Phys. Hung. A 22, 271 (2005) [nucl-th/0406066]; J. Phys. G 30, S1239 (2004).

18. B. Muller, R. J. Fries and S. A. Bass, Phys. Lett. B 618, 77 (2005).

19. C. B. Dover et al. Phys. Rev. C 44 (1991) 1636.

20. R. Scheibl and U. W. Heinz, Phys. Rev. C 59 (1999) 1585. 
21. R. J. Fries, S. A. Bass and B. Muller, Phys. Rev. Lett. 94, 122301 (2005).

22. X. N. Wang, M. Gyulassy and M. Plumer, Phys. Rev. D 51 (1995) 3436; R. Baier et al., Nucl. Phys. B 483 (1997) 291; M. Gyulassy, P. Levai and I. Vitev, Nucl. Phys. B 571 (2000) 197; U. A. Wiedemann, Nucl. Phys. A 690 (2001) 731.

23. V. Greco (2004), private communication.

24. D. Molnar, nucl-th/0408044.

25. B. Zhang, Comput. Phys. Commun. 109 (1998) 193.

26. B. Zhang, M. Gyulassy and C. M. Ko, Phys. Lett. B455, 45 (1999).

27. D. Molnar and M. Gyulassy, Phys. Rev. C 62 (2000) 054907.

28. D. Molnar and M. Gyulassy, Nucl. Phys. A697, 495 (2002), A703, 893(E) (2002); ibid. A698, 379 (2002).

29. D. Molnar and P. Huovinen, Phys. Rev. Lett. 94, 012302 (2005).

30. D. Molnar, J. Phys. G 31, S421 (2005).

31. D. Molnár, Nucl. Phys. A661, 236c (1999).

32. Z. Xu and C. Greiner, Phys. Rev. C 71, 064901 (2005); hep-ph/0509324.

33. S. A. Bass, B. Muller and D. K. Srivastava, Phys. Lett. B 551, 277 (2003).

34. C. Adler et al. [STAR Collaboration], Phys. Rev. Lett. 90, 032301 (2003); K. Filimonov [STAR Collaboration], Nucl. Phys. A715, 737 (2003).

35. K. Adcox et al. [PHENIX Collaboration], Phys. Rev. Lett. 89, 212301 (2002);

N. N. Ajitanand [PHENIX Collaboration], Nucl. Phys. A 715, 765 (2003).

36. B. B. Back et al. [Phobos Collaboration], nucl-ex/0407012.

37. P. Danielewicz and M. Gyulassy, Phys. Rev. D 31, 53 (1985).

38. G. Policastro, D. T. Son and A. O. Starinets, Phys. Rev. Lett. 87, 081601 (2001); P. Kovtun, D. T. Son and A. O. Starinets, Phys. Rev. Lett. 94, 111601 (2005).

39. D. Teaney, Nucl. Phys. A715, 817 (2003); Phys. Rev. C68, 034913 (2003).

40. D. Molnar, MPC 1.6.7. This parton transport code can be downloaded from the WWW at http://www-cunuke.phys.columbia.edu/people/molnard .

41. B. Zhang, L. W. Chen and C. M. Ko, Phys. Rev. C 72, 024906 (2005); nuclth/0509095.

42. G. D. Moore and D. Teaney, Phys. Rev. C 71, 064904 (2005).

43. F. Laue [STAR Collaboration], J. Phys. G 31 (2005) S1121.

44. S. S. Adler et al. [PHENIX Collaboration], Phys. Rev. C 72, 024901 (2005).

45. V. Greco, C. M. Ko and R. Rapp, Phys. Lett. B 595, 202 (2004).

46. D. Molnar, nucl-th/0503051.

47. U. Heinz and P. F. Kolb, Phys. Lett. B 542, 216 (2002).

48. S. Soff, S. A. Bass and A. Dumitru, Phys. Rev. Lett. 86 (2001) 3981.

49. D. Molnar and M. Gyulassy, Phys. Rev. Lett. 92 (2004) 052301.

50. Z. w. Lin, C. M. Ko and S. Pal, Phys. Rev. Lett. 89 (2002) 152301.

51. P. Huovinen et al., Phys. Lett. B 503, 58 (2001).

52. S. Pratt and S. Pal, Nucl. Phys. A 749, 268 (2005) [Phys. Rev. C 71, 014905 (2005)]

53. V. Greco and C. M. Ko, nucl-th/0505061.

54. M. Gyulassy, K. Frankel and E. a. Remler, Nucl. Phys. A 402 (1983) 596.

55. J. L. Nagle et al., Phys. Rev. C 53 (1996) 367. 